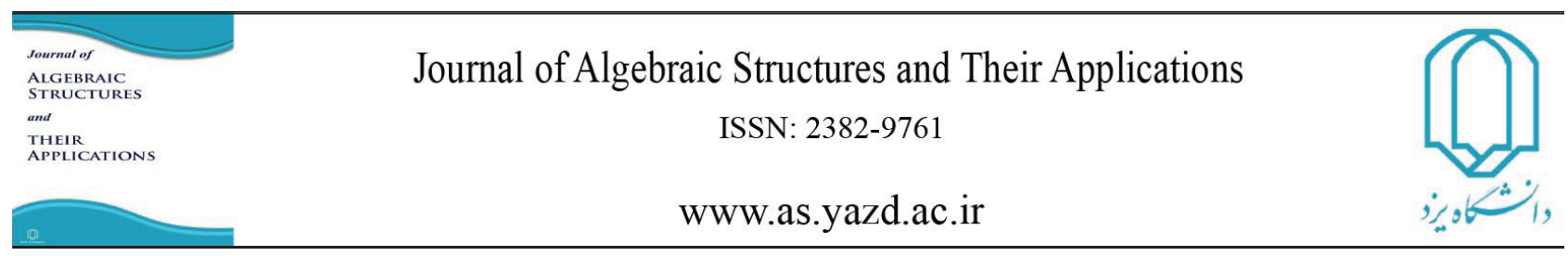

Algebraic Structures and Their Applications Vol. 5 No. 1 ( 2018 ) pp 1-21.

\title{
AN INVESTIGATION ON REGULAR RELATIONS OF UNIVERSAL HYPERALGEBRAS
}

\author{
S. RASOULI AND B. DAVVAZ*
}

Communicated by R. Borzooei

\begin{abstract}
In this paper, by considering the notion of $\Sigma$-hyperalgebras for an arbitrary signature $\Sigma$, we study the notions of regular and strongly regular relations on a $\Sigma$-hyperalgebra, $\mathfrak{A}$. We show that each regular relation which contains a strongly regular relation is a strongly regular relation. Then we concentrate on the connection between the fundamental relation of $\mathfrak{A}$ and the set of complete parts of $\mathfrak{A}$.
\end{abstract}

\section{INTRODUCTION}

The theory of hypergroups was introduced by F. Marty in 1934,(see [18]). Hyperalgebras (or multialgebras) are particular relational systems which generalize the concept of universal algebras. The hyperstructure theory and its applications so far, have been investigated by many mathematicians in various fields, for example in graphs and hypergraphs theory [2], in category theory [11, 22] and in n-ary hyperalgebras [5, 16]. The recent book [4] contains wealthy

DOI:http://dx.doi.org/10.29252/asta.5.1.1

MSC(2010): 0N20, 08A05, 08A30, 03C05.

Keywords: Universal algebras, Regular relation, Fundamental relation, Complete part.

Received: 04 May 2017, Accepted: 24 July 2018.

*Corresponding author

(C) 2018 Yazd University. 
applications. There are also applications to the following subjects: geometry, hypergraphs, binary relations, combinatorics, codes, cryptography, probability, etc.

In 1962, G. Grätzer began to study hyperalgebras [II] and it was followed by H. E. Pickett [23] who investigated homomorphisms and subalgebras of hyperalgebras. Then H. Höft and P. E. Howard [14] and G. E. Hansoul [13] studied hyperalgebras. Later, D. Schweigert in [30] studied the congruences of hyperalgebras and the exponentiations of universal hyperalgebras. The concepts of regular relations, quotient hyperalgebras and homomorphisms are all closely related. In the hyperstructure theory, regular relations play the same role as what congruences do in theory of universal algebras. Furthermore, they provide us new hyperstructures on the quotient sets. Also, an especial type of regular relations so called strongly regular relations provide a connection between the hyperstructure theory and universal algebras. In particular, the fundamental relation as the smallest strongly regular relation is an important tool in the hyperstructure theory. According to [2T], the factorization of a hyperalgebra modulo its fundamental relation provides a functor into the category of universal algebras. C. Pelea studied the fundamental relations of a hyperalgebras [20] and investigated them on the universal algebras point of view in [21, [22]. Also, M. M. Ebrahimi et al. in [10] studied the quotient and Isomorphism Theorems of Universal Hyperalgebras. Thus it seems adequate to undertake a study of hyperalgebras in the spirit of universal algebras.

On the other hand, the concept of complete parts and fundamental relations are closely related. Complete parts were introduced and studied by M. Koskas in [15]. Later, this topic was analyzed by P. Corsini in [Z] and Y. Sureau in [3] mostly in the general theory of hypergroups. M. De Salvo in [ [7], studied complete parts from a combinatorial point of view. A generalization of them, called $n$-complete parts, was introduced by R. Migliorato in [1.9]. Other hypergroupists gave a contribution to the study of complete parts and of the heart of a hypergroup. Among them, V. Leoreanu analyzed the structure of the heart of a hypergroup in [I7].

In this paper, we study the concept of regular and strongly regular relations. The paper is organized in six sections. In Section 2, we gather the definitions and basic properties of hyperalgebras and universal algebras which will be used in the next sections. In Section 3 , we concentrate on the lattice structure of regular and strongly regular relations of a $\Sigma$ hyperalgebra $\mathfrak{A}$. In Section 4, the behavior of the regular and strongly regular relations under homomorphisms are investigated and then, we state and prove the hyperstructure's version of Isomorphism Theorems and Correspondence Theorem. In Section 5, we introduce the notion of complete parts for an arbitrary $\Sigma$-hyperalgebras and study their properties. Also, we find some conditions with respect to complete parts for transitivity of the relation $f_{\mathfrak{A}}$. Section 6 is a conclusion. 


\section{A BRIEF EXCURSION INTO UNIVERSAL HYPERALGEBRAS}

This section contains a survey of the basic elements of universal algebras and hyperalgebras which will be used in the next sections. In fact, we explain what is meant by a hyperalgebra and then give several examples of familiar hyperalgebras. These examples show that different hyperalgebras may have several common properties. This observation provides a motivation for the study of $\Sigma$-hyperalgebras.

In the sequel $A$ is a fixed nonempty set, $\mathcal{P}^{*}(A)$ is the family of all nonempty subsets of $A$, $w$ is the set of positive integers and for $n \in w$ we denote the set of $n$-tuples over $A$, By $A^{n}$. Also, by $B \subseteq_{w} A$ we mean that $B$ is a finite subset of $A$ and $\{1, \cdots, n\}$ is denoted by $I_{n}$.

Let $A$ be a nonempty set. A family $\mathcal{C}$ of subsets of $A$ that is closed under the intersection of arbitrary subfamilies is called a closed-set system over $A$. If $\mathcal{C}$ is also closed under the union of subfamilies that are upward directed under inclusion, then it is called an algebraic closed-set system. An algebraic closed set system $\mathcal{C}$ forms an algebraic lattice $(\mathcal{C}, \cap, \vee)$ under set-theoretic inclusion. The closure operator associated with a given closed set system $\mathcal{C}$ is denoted by $C l_{\mathcal{C}}$. Also, for any comparable elements $x, y$ of the poset $P$ where $x \leq y$, let $[x, y]_{P}=\{z \in P \mid x \leq z \leq y\}$. Let $P$ be a poset and $x, y$ be any comparable elements of $P$. We say $x$ is covered by $y$ if $[x, y]_{P}=\{x, y\}$. The set of all elements of $P$ which covered by $y$ is denoted by $\operatorname{Cob}(y)$.

An $n$-ary hyperoperation (or function) on $A$ is a function $\sigma$ from $A^{n}$ to $\mathcal{P}^{*}(A) ; n$ is the arity (or rank) of $\sigma$. A finitary hyperoperation is an $n$-ary hyperoperation, for some $n$. The image of $\left(a_{1}, \cdots, a_{n}\right)$ under an $n$-ary hyperoperation $\sigma$ is denoted by $\sigma\left(a_{1}, \cdots, a_{n}\right)$. A hyperoperation $\sigma$ on $\mathrm{A}$ is called a nullary hyperoperation (or constant) if its arity is zero; in fact, A nullary hyperoperation on $A$ is just an element of $\mathcal{P}^{*}(A)$; i.e. a non void subset of $A$. A hyperoperation $\sigma$ on $A$ is unary, binary or ternary if its arity is 1,2 or 3 , respectively.

A signature or language type is a set $\Sigma$ together with a mapping $\rho: \Sigma \longrightarrow w$. The elements of $\Sigma$ are called hyperoperation symbols. For each $\sigma \in \Sigma, \rho(\sigma)$ is called the arity or rank of $\sigma$. In the sequel, for each $n \in w, \Sigma_{n}=\{\sigma \mid \rho(\sigma)=n\}$.

Let $\Sigma$ be a signature. A $\Sigma$-hyperalgebraic structure is an ordered couple $\mathfrak{A}=\left(A,<\sigma^{\mathfrak{A}}\right.$ : $\sigma \in \Sigma>$ ), where $A$ is a nonempty set and $\sigma^{\mathfrak{A}}$ is a function from $A^{\rho(\sigma)}$ to $\mathcal{P}^{*}(A)$, for all $\sigma \in \Sigma$. The set $A$ is called the universe (or underlying set) of $\mathfrak{A}$ and the $\sigma^{\mathfrak{A}}$ 's are called the fundamental hyperoperations of $\mathfrak{A}$. In the following, we prefer to write just $\sigma$ for $\sigma^{\mathfrak{A}}$ if this convention creates an ambiguity which seldom causes a problem.

In this paper we shall use the following abbreviated notations: the sequences $x_{i}, \cdots, x_{j}$ and $\left(x_{i}, y_{i}\right), \cdots,\left(x_{j}, y_{j}\right)$ will be denoted by $x_{i}^{j}$ and $(x, y)_{i}^{j}$, respectively. For $j<i$ is the empty symbol. In this convention

$$
\sigma^{\mathfrak{A}}\left(x_{1}, \cdots, x_{i}, y_{i+1}, \cdots, y_{j}, z_{j+1}, \cdots, z_{\rho(\sigma)}\right)
$$


will be written as $\sigma^{\mathfrak{A}}\left(x_{1}^{i}, y_{i+1}^{j}, z_{j+1}^{\rho(\sigma)}\right)$. In this case when $y_{i+1}=\cdots=y_{j}=y$ the last expression will be written in the form $\sigma^{\mathfrak{A}}\left(x_{1}^{i},{ }^{(j-i)} y, z_{j+1}^{\rho(\sigma)}\right)$. Similarly, for subsets $B_{1}^{\rho(\sigma)}$ of $A$ we define

$$
\sigma^{\mathfrak{A}}\left(B_{1}^{\rho(\sigma)}\right)=\cup\left\{\sigma^{\mathfrak{A}}\left(b_{1}^{\rho(\sigma)}\right) \mid b_{i} \in B_{i}, \forall i \in I_{\rho(\sigma)}\right\} .
$$

Also, for each $i \in I_{\rho(\sigma)}$ and $a_{1}^{\rho(\sigma)} \in A$ we define

$$
\sigma_{i}^{\mathfrak{A}}\left(a_{1}^{\rho(\sigma)}\right)=\left\{z \in A: a_{i} \in \sigma^{\mathfrak{A}}\left(a_{1}^{i-1}, z, a_{i+1}^{\rho(\sigma)}\right)\right\}
$$

A hyperalgebra $\mathfrak{A}$ is unary if all hyperoperations are unary and it is mono-unary if it has just one unary hyperoperation. $\mathfrak{A}$ is a hypergroupoid if it has just one binary hyperoperation $\sigma$. According to [[] $], \Sigma$-hyperalgebra $\mathfrak{A}$ is called an n-ary hypergroupoid if $\Sigma=\{\sigma\}$ and $\rho(\sigma)=n$. If $\sigma$ is a binary hyperoperation, we write $a \sigma b$ for the image of $(a, b)$ under $\sigma$. A hyperalgebra $\mathfrak{A}$ is finite if $|A|$ is finite. Let $\mathfrak{A}$ be a $\Sigma$-hyperalgebra. Hyperoperation $\sigma^{\mathfrak{A}}$ is called an operation, Without any ambiguity, if for any $a_{1}^{\rho(\sigma)} \in A$, we have $\left|\sigma^{\mathfrak{A}}\left(a_{1}^{\rho(\sigma)}\right)\right|=1$. $\mathfrak{A}$ is called a $\Sigma$-algebra if for any $\sigma \in \Sigma, \sigma^{\mathfrak{A}}$ is an operation.

Let $\mathfrak{A}=\left(A,<\sigma^{\mathfrak{A}}: \sigma \in \Sigma>\right)$ be a $\Sigma$-hyperalgebra and $\sigma \in \Sigma$. Now, we extend an $n$-ary hyperoperation $\sigma^{\mathfrak{A}}$ to an $n$-ary operation $\sigma^{\mathfrak{P}_{\mathfrak{A}}}$ on $\mathcal{P}^{*}(A)$ by setting for all $A_{1}^{\rho(\sigma)} \in \mathcal{P}^{*}(A)$

$$
\left.\sigma^{\mathfrak{P}_{\mathfrak{A}}}\left(A_{1}^{\rho(\sigma)}\right)=\bigcup\left\{\sigma^{\mathfrak{A}}\left(a_{1}^{\rho(\sigma)}\right) \mid a_{i} \in A_{i}, i \in I_{\rho(\sigma)}\right)\right\} .
$$

It is easy to see that $\mathfrak{P}_{\mathfrak{A}}=\left(\mathcal{P}^{*}(A),<\sigma^{\mathfrak{P}_{\mathfrak{A}}}: \sigma \in \Sigma>\right)$ is a $\Sigma$-algebra.

Definition 2.1. [5] Let $\mathfrak{A}$ be a $\Sigma$-hyperalgebra, $\sigma \in \Sigma$ and $j<i<\rho(\sigma)$. We say that $\sigma$ is weakly $(i, j)$-associative if for each $a_{1}^{2 \rho(\sigma)-1} \in A$ we have

$$
\sigma^{\mathfrak{A}}\left(a_{1}^{i-1}, \sigma^{\mathfrak{A}}\left(a_{i}^{\rho(\sigma)+i-1}\right), a_{\rho(\sigma)+i}^{2 \rho(\sigma)-1}\right) \cap \sigma^{\mathfrak{A}}\left(a_{1}^{j-1}, \sigma^{\mathfrak{A}}\left(a_{j}^{\rho(\sigma)+j-1}\right), a_{\rho(\sigma)+j}^{2 \rho(\sigma)-1}\right) \neq \emptyset,
$$

and $(i, j)$-associative if

$$
\sigma^{\mathfrak{A}}\left(a_{1}^{i-1}, \sigma^{\mathfrak{A}}\left(a_{i}^{\rho(\sigma)+i-1}\right), a_{\rho(\sigma)+i}^{2 \rho(\sigma)-1}\right)=\sigma^{\mathfrak{A}}\left(a_{1}^{j-1}, \sigma^{\mathfrak{A}}\left(a_{j}^{\rho(\sigma)+j-1}\right), a_{\rho(\sigma)+j}^{2 \rho(\sigma)-1}\right) .
$$

We say that $\sigma$ is (weakly) associative if it is (weakly) $(i, j)$-associative for each $i, j \in I_{\rho(\sigma)}$.

Definition 2.$]$ is a generalization of associative binary hyperoperation. An $n$-ary hypergroupoid $\mathfrak{A}$ is called an $n$-ary semihypergroup ( $n$-ary $H_{v}$-semigroup) if $\sigma$ is (weakly) associative.

Example 2.2. (i) [6] Let $A=\{a, b, c\}$ with the hyperoperation $\circ$ defined by the following table

\begin{tabular}{c|ccc}
$\circ$ & $a$ & $b$ & $c$ \\
\hline$a$ & $a$ & $b$ & $c$ \\
$b$ & $b$ & $\{a, c\}$ & $\{a, b\}$ \\
$c$ & $c$ & $\{a, b\}$ & $\{a, b\}$
\end{tabular}


Now, we define the ternary hyperoperation $\sigma$ on $A$ by $\sigma(x, y, z)=x \circ y \circ z$, for each $x, y, z \in A$. One can see that $\mathfrak{A}=(A, \sigma)$ is a 3-ary semihypergroup.

(ii) Let $A=\{a, b, c\}$ with the hyperoperation $\circ$ defined by the following table

\begin{tabular}{c|ccc}
$\circ$ & $a$ & $b$ & $c$ \\
\hline$a$ & $a$ & $b$ & $c$ \\
$b$ & $b$ & $c$ & $\{a, b\}$ \\
$c$ & $c$ & $b$ & $\{a, b\}$
\end{tabular}

It is easy to check that $\mathfrak{A}=(A, \circ)$ is a $H_{v^{-}}$semigroup but it is not a semihypergroup.

Definition 2.3. [5, 6] Let $\mathfrak{A}$ be a $\Sigma$-hyperalgebra, $\sigma \in \Sigma$ and $B \subseteq A$. We say that $B$ has the reproduction axiom with respect to $\sigma$ if for each $b_{1}^{i-1}, b, b_{i+1}^{\rho(\sigma)} \in B$ and $1 \leq i \leq \rho(\sigma)$ the relation

$$
b \in \sigma_{i}^{\mathfrak{A}}\left(b_{1}^{i-1}, x, b_{i+1}^{\rho(\sigma)}\right),
$$

has a solution $x \in B$. Observe that condition (1) can be reformulated as follows

$$
B=\sigma^{\mathfrak{A}}\left(b_{1}^{i-1}, B, b_{i+1}^{\rho(\sigma)-i}\right) .
$$

An $n$-ary semihypergroup ( $H_{v}$-semigroup) $\mathfrak{A}$ is called an $n$-ary hypergroup ( $H_{v}$-group) if $A$ satisfies the reproduction axiom. An $n$-ary hypergropouid which satisfies the reproduction axiom is called $n$-ary hyperquasigroup.

Example 2.4. [6] Let $A=\{a, b, c\}$. We define hyperoperation $\sigma$ as follows

$$
\sigma(x, y, z)= \begin{cases}x & x=y=z, \\ b & x \neq y \neq z, \\ z & x=y, x \neq z, x \neq b, \\ \{a, c\} & x=y=b, z \neq b .\end{cases}
$$

One can see that $\mathfrak{A}=\{A, \sigma\}$ is a 3-ary hypergroup.

One can find definitions of hyperlattices in [24, 25], hyper MV-algebras in [27, 26], hyper BCK-algebras in [28] and etc.

Let $\mathfrak{A}$ and $\mathfrak{B}$ be $\Sigma$-hyperalgebras. A map $f: A \longrightarrow B$ is a homomorphism (resp. good homomorphism), in symbols $f: \mathfrak{A} \longrightarrow \mathfrak{B}$, if for all $\sigma \in \Sigma$ and all $a_{1}^{\rho(\sigma)} \in A$,

$$
\begin{gathered}
\left.f\left(\sigma^{\mathfrak{A}}\left(a_{1}^{\rho(\sigma)}\right)\right) \subseteq \sigma^{\mathfrak{B}}\left(f\left(a_{1}\right), \cdots, f\left(a_{\rho(\sigma)}\right)\right)\right), \\
\left(\text { resp. } f\left(\sigma^{\mathfrak{A}}\left(a_{1}^{\rho(\sigma)}\right)\right)=\sigma^{\mathfrak{B}}\left(f\left(a_{1}\right), \cdots, f\left(a_{\rho(\sigma)}\right)\right) .\right.
\end{gathered}
$$

$\operatorname{Hom}(\mathfrak{A}, \mathfrak{B})$ will denote the set of all homomorphisms and $\operatorname{Hom}_{G}(\mathfrak{A}, \mathfrak{B})$ will denote the set of all good homomorphisms from $\mathfrak{A}$ to $\mathfrak{B}$. $f: \mathfrak{A} \longrightarrow \mathfrak{B}$ is called an isomorphism if 
$f \in \operatorname{Hom}(\mathfrak{A}, \mathfrak{B})$ and $f^{-1} \in \operatorname{Hom}(\mathfrak{B}, \mathfrak{A})$, too. As it results from [[2:3], the isomorphisms can be characterized as being the bijective good homomorphisms.

Example 2.5. [26] Let $M=\{0, a, b, 1\}$. Consider the following tables:

\begin{tabular}{c|cccc}
$\oplus$ & 0 & $a$ & $b$ & 1 \\
\hline 0 & $\{0\}$ & $\{a\}$ & $\{b\}$ & $\{1\}$ \\
$a$ & $\{a\}$ & $\{b\}$ & $\{1\}$ & $\{1\}$ \\
$b$ & $\{b\}$ & $\{1\}$ & $\{1\}$ & $\{1\}$ \\
1 & $\{1\}$ & $\{1\}$ & $\{1\}$ & $\{1\}$
\end{tabular}

\begin{tabular}{l|llll}
$*$ & 0 & $\mathrm{a}$ & $\mathrm{b}$ & 1 \\
\hline & 1 & $\mathrm{~b}$ & $\mathrm{a}$ & 0
\end{tabular}

It is easy to see that $\mathfrak{M}=\left(M, \oplus,{ }^{*}, 0\right)$ is an MV-algebra which is not totally ordered. Also, let $H S_{3}=\left(S_{3}, \oplus,{ }^{*}, 0\right)$ is a hyper MV-algebra where $S_{3}=\left\{0, \frac{1}{3}, \frac{2}{3}, 1\right\}, x \oplus y:=[0, \min (1, x+y)] \cap S_{3}$ and $x^{*}=1-x$. Now, let $f: \mathfrak{M} \longrightarrow H S_{3}$ be a map that $f(0)=0, f(a)=\frac{1}{3}, f(b)=\frac{2}{3}$ and $f(1)=1$. It is easy to check that $f$ is a hyper MV-algebra homomorphism. For example $f(a \oplus a)=f(\{b\})=\left\{\frac{2}{3}\right\}$ and $f(a) \oplus f(a)=\frac{1}{3} \oplus_{H 3} \frac{1}{3}=\left\{0, \frac{1}{3}, \frac{2}{3}\right\}$ so $f(a \oplus a) \subsetneq f(a) \oplus f(a)$. Also, if we define $g: H S_{3} \longrightarrow \mathfrak{M}$ such that $g(0)=0, g\left(\frac{1}{3}\right)=a, g\left(\frac{2}{3}\right)=b$ and $g(1)=1$, then $g$ is a dual homomorphism that is not a homomorphism, since $g\left(\frac{1}{3} \oplus_{H 3} \frac{1}{3}\right)=\{0, a, b\}$ but $g\left(\frac{1}{3}\right) \oplus g\left(\frac{1}{3}\right)=\{b\}$.

The concepts of regular relation, quotient hyperalgebra, and homomorphism are all closely related. Recall that a binary relation on $A$ is a subset of $A^{2}$. If $\theta, \varphi$ are two binary relations, the relational product $\theta$ and $\varphi$, denoted by $\theta \circ \varphi$, is defined as a binary relation on $A$ by

$$
\theta \circ \varphi=\left\{(x, y) \in A^{2}: \exists z \in A \text {, such that }(x, z) \in \theta,(z, y) \in \varphi\right\}
$$

The notion of $\theta^{n}$ is reserved for $\theta \circ \cdots \circ \theta$, (n-times). An equivalence relation is a binary relation which satisfies reflexivity, symmetry and transitivity conditions. The diagonal relation $\Delta_{A}$ on $A$ is the set $\{(x, x): x \in A\}$ and the total relation $\nabla_{A}$ is the set $\{(x, y): x, y \in A\}$. In the following, the set of all transitive relations and equivalence relations on $A$ is denoted by $\operatorname{Tr}(A)$ and $E q(A)$, respectively. If $\Re \in E q(A)$ and $X \subseteq A$, then the equivalence class of $x \in A$ and the set of all equivalence classes of $x \in X$ are denoted by $x / \Re$ and $X / \Re$, respectively. Clearly, $\Delta_{A}, \nabla_{A} \in E q(A)$. Now, we give a proposition which its proof can be found in elementary universal algebras books.

Remark 2.6. Let $A$ be a nonempty set. Then $\operatorname{Tr}(A)$ is an algebraic closed set system. The closure operator associated with $\operatorname{Tr}(A)$ is denoted by $\operatorname{Tr}$. If $\theta \subseteq A^{2}$, then $\operatorname{Tr}(\theta)=\cup\left\{\theta^{n}: n \in\right.$ $w\}$. Furthermore, if $\left\{\theta_{i}\right\}_{i \in I}$ is a family of subsets of $\operatorname{Tr}(A)$ then

$$
\vee_{i \in I} \theta_{i}=\operatorname{Tr}\left(\cup \theta_{i}\right)=\cup\left\{\theta_{i_{0}} \circ \cdots \circ \theta_{i_{n}}: n \in w, i_{0}, \cdots, i_{n} \in I\right\}
$$


Also, $(E q(A), \subseteq)$ is a complete sublattice of $(\operatorname{Tr}(A), \subseteq)$. Let $E q: \mathcal{P}\left(A^{2}\right) \longrightarrow \mathcal{P}\left(A^{2}\right)$ be the closure operator associated with $E q(A)$. If $\theta \in \mathcal{P}\left(A^{2}\right)$, then one can see that

$$
E q(\theta)=\Delta \cup \operatorname{Tr}\left(\theta^{-1} \cup \theta\right)
$$

Thus if $\theta$ is reflexive and symmetric we obtain that $E q(\theta)=\operatorname{Tr}(\theta)$.

Let $\Re$ be a binary relation on a nonempty set $A$. We can extend $\Re$ on $\mathcal{P}^{*}(A)$, in the following ways. Let $X, Y \in \mathcal{P}^{*}(A)$. then we write

(i) $X \bar{\Re} Y$ if for all $x \in X$, there exists $y \in Y$ such that $x \Re y$, and vice versa.

(ii) $X \overline{\bar{\Re}} Y$ if for all $x \in X$ and $y \in Y, x \Re y$.

Lemma 2.7. Let $A$ be a nonempty set, $\left\{X_{i}\right\}_{i=1}^{n+1} \subseteq \mathcal{P}^{*}(A)$ and $\Re_{1}^{n}$ be binary relations such that $X_{i} \overline{\Re_{i}} X_{i+1}$, for each $i \in I_{n+1}$. Then $X_{1} \overline{\circ_{i=1}^{n} \Re_{i}} X_{n+1}$. Furthermore, if $X_{j} \overline{\overline{\Re_{j}}} X_{j+1}$, for some $j \in\{1, \cdots, n+1\}$ then $X_{1} \overline{\overline{\mathrm{o}_{i=1}^{n} \Re_{i}}} X_{n+1}$.

Proof. First let $X_{i} \overline{\Re_{i}} X_{i+1}$, for each $i \in I_{n+1}$. Assume that $x_{1} \in X_{1}$. So there exists $x_{i} \in X_{i}$, for each $i \in\{2, \cdots, n+1\}$ such that $x_{1} \Re_{1} x_{2}$ and $x_{i} \Re_{i} x_{i+1}$. Hence, $\left(x_{1}, x_{n+1}\right) \in \circ_{i=1}^{n} \Re_{i}$ and this holds the result. Now, let $X_{i} \overline{\Re_{i}} X_{i+1}$, for each $i \in I_{n+1}$ and $X_{i} \overline{\overline{\Re_{i}}} X_{i+1}$, for some $j \in I_{n+1}$. Assume that $x_{1} \in X_{1}$ and $x_{n} \in X_{n}$. So there exist $x_{j} \in X_{j}$ and $x_{j+1} \in X_{j+1}$ such that $\left(x_{1}, x_{j}\right) \in \Re_{1} \circ \cdots \circ \Re_{j-1},\left(x_{j+1}, x_{n}\right) \in \Re_{j+1} \circ \cdots \circ \Re_{n}$ and $\left(x_{j}, x_{j+1}\right) \in \Re_{j}$. It implies that $\left(x_{1}, x_{n}\right) \in \Re_{1} \circ \cdots \circ \Re_{n}$ and it gets the result.

Clearly, if $\Re$ is a transitive relation on a nonempty set $A$ then $X \bar{\Re} Y$ and $Y \bar{\Re} Z$, for some $X, Y, Z \subseteq A$, imply $X \bar{\Re} Z$.

Definition 2.8. Let $\mathfrak{A}=\left(A,\left\langle\sigma^{\mathfrak{A}}: \sigma \in \Sigma>\right)\right.$ be a $\Sigma$-hyperalgebra, $\Re$ be a binary relation on $A, \sigma \in \Sigma$ and $\delta=\left(\delta_{1}, \cdots, \delta_{\rho(\sigma)}\right)$ such that $\delta_{i} \in\{0,1\}$. Let $\delta^{1}=\left\{i \in w: \delta_{i}=1\right\}$. Then we say that $\Re$ has the regular (resp. strongly regular) substitution property of type $\delta$ with respect to $\sigma$ if for each $a_{1}^{\rho(\sigma)}, b_{1}^{\rho(\sigma)} \in A$, the following condition holds:

$$
\begin{gathered}
a_{i} \Re b_{i}, \forall i \in \delta^{1}, \text { implies } \sigma^{\mathfrak{A}}\left(a_{1}^{\rho(\sigma)}\right) \bar{\Re} \sigma^{\mathfrak{A}}\left(b_{1}^{\rho(\sigma)}\right) \\
\left(\text { resp. } a_{i} \Re b_{i}, \forall i \in \delta^{1}, \text { implies } \sigma^{\mathfrak{A}}\left(a_{1}^{\rho(\sigma)}\right) \overline{\bar{\Re}} \sigma^{\mathfrak{A}}\left(b_{1}^{\rho(\sigma)}\right)\right)
\end{gathered}
$$

$\Re$ has the regular (resp. strongly regular) substitution property with respect to $\sigma$ if $\left|\delta^{1}\right|=$ $\rho(\sigma)$. Also, $\Re$ has the regular (resp. strongly regular) substitution property on $\Sigma$-hyperalgebra $\mathfrak{A}$, if $\Re$ has the regular (resp. strongly regular) substitution property with respect to each $\sigma \in \Sigma$.

Lemma 2.9. Let $\mathfrak{A}$ be a $\Sigma$-hyperalgebra and $\mathcal{R}=\left\{\Re_{i}\right\}_{i \in I}$ be a family of binary relations on $A$ with the regular (resp. strongly regular) substitution property. Then for each $\sigma \in \Sigma$ the binary 
relation $\operatorname{Tr}(\cup \mathcal{R})$ has the regular (resp. strongly regular) substitution property with respect to $\sigma$. In particular, $E q(\cup \mathcal{R})$ has the regular (resp. strongly regular) substitution property.

Proof. Let $\sigma \in \Sigma$ and $(a, b)_{1}^{\rho(\sigma)} \in \operatorname{Tr}(\cup \mathcal{R})$, for $i \in I_{\rho(\sigma)}$. So by [3], for each $i \in I_{\rho(\sigma)}$ there exist an integer $n_{i}$ such that $\left(a_{i}, b_{i}\right) \in \circ_{j=1}^{n_{i}} \Re_{m_{i j}}$, where $m_{i 1}, \cdots, m_{i n_{i}} \in I$. Thus for each $i \in I_{\rho(\sigma)}$ there are $c_{i 1}, \cdots, c_{i\left(n_{i}+1\right)} \in A$ such that

$$
a_{i}=c_{i 1} \Re_{m_{i 1}} c_{i_{2}} \cdots c_{i n_{i}} \Re_{m_{i n_{i}}} c_{i\left(n_{i}+1\right)}=b_{i} .
$$

Now, by the regular substitution property and Lemma [2.7, for each $i \in I_{\rho(\sigma)}$ we have

$$
\left(\sigma^{\mathfrak{A}}\left(b_{1}^{i-1}, a_{i}^{\rho(\sigma)}\right), \sigma^{\mathfrak{A}}\left(b_{1}^{i}, a_{i+1}^{\rho(\sigma)}\right)\right) \in \overline{\mathrm{o}_{j=1}^{n_{i}} \Re_{m_{i j}}}
$$

and it implies that

$$
\left(\sigma^{\mathfrak{A}}\left(a_{1}^{\rho(\sigma)}\right), \sigma^{\mathfrak{A}}\left(b_{1}^{\rho(\sigma)}\right)\right) \in \overline{\circ_{i=1}^{n} \circ_{j=1}^{n_{i}} \Re_{m_{i j}}} \subseteq \overline{\operatorname{Tr}(\cup \mathcal{R})} .
$$

Also, by equation $\mathbb{Q}$, we can see that $E q(\cup \mathcal{R})$ has the regular (resp. strongly regular) substitution property.

\section{The lattice Structure of Regular Relations}

In this section, we introduce the notion of regular and strongly regular relations and concentrate on the lattice structures of them.

Definition 3.1. Let $\mathfrak{A}$ be a $\Sigma$-hyperalgebra and $\Re \in E q(\mathfrak{A})$. $\Re$ is called a regular (resp. strongly regular) relation on $\mathfrak{A}$ if it has the regular (resp. strongly regular) substitution property.

The set of regular relations on $\mathfrak{A}$ is denoted by $\operatorname{Reg}(\mathfrak{A})$ and the set of strongly regular relations on $\mathfrak{A}$ is denoted by $\operatorname{Reg}_{s}(\mathfrak{A})$. Clearly, $\operatorname{Re} g_{s}(\mathfrak{A}) \subseteq \operatorname{Reg}(\mathfrak{A})$. Also, $\mathbf{B}(\operatorname{Reg}(\mathfrak{A}))$ is reserved for the difference between $\operatorname{Reg}(\mathfrak{A})$ and $\operatorname{Reg}_{s}(\mathfrak{A})$.

Remark 3.2. (1) $\Delta_{\mathfrak{A}} \in \operatorname{Reg}(\mathfrak{A})$ and $\nabla_{\mathfrak{A}} \in \operatorname{Reg}_{s}(\mathfrak{A})$.

(2) if $\mathfrak{A}$ is an algebra then $\mathbf{B}(\operatorname{Reg}(\mathfrak{A}))=\emptyset$ and $\operatorname{Reg}(\mathfrak{A})=\operatorname{Con}(\mathfrak{A})$ where $\operatorname{Con}(\mathfrak{A})$ is the set of congruence of $\mathfrak{A}$.

According to [I0], $\operatorname{Reg}(\mathfrak{A})$ is not even closed under finite intersection. But, $(\operatorname{Reg}(\mathfrak{A}), \subseteq)$ is a complete $\vee$-semilattice in which joins are calculated the same as when working with equivalence relations. Therefore $(\operatorname{Reg}(\mathfrak{A}), \subseteq)$ is a complete lattice with the meet operation defined by

$$
\bigwedge_{i \in I} \Re_{i}=\bigvee\left\{\Re \in \operatorname{Reg}(\mathfrak{A}) \mid \Re \subseteq \bigcap_{i \in I} \Re_{i}\right\}=\operatorname{Tr}\left(\bigcup\left\{\Re \in \operatorname{Reg}(\mathfrak{A}) \mid \Re \subseteq \bigcap_{i \in I} \Re_{i}\right\}\right)
$$

Theorem 3.3. $\left(\operatorname{Reg}_{s}(\mathfrak{A}), \subseteq\right)$ is a complete sublattice of $(E q(A), \subseteq)$. 
Proof. To verify that $\operatorname{Reg}_{s}(\mathfrak{A})$ is closed under arbitrary intersection is straightforward. Now, for arbitrary joins in $\operatorname{Reg}_{s}(\mathfrak{A})$, let $\left\{\Re_{i}\right\}_{i \in I}$ be a nonempty family of $\operatorname{Reg}_{s}(\mathfrak{A}), \sigma \in \Sigma$ and $(a, b)_{1}^{\rho(\sigma)} \in \vee_{i \in I} \Re_{i}$ where $\vee$ is the join of $E q(A)$, then by Remark [2.6], for each $i \in I_{\rho(\sigma)}$ there exists $J_{i}=\left\{i_{0}, \cdots, i_{m_{i}}\right\} \subseteq_{w} I$ such that $\left(a_{i}, b_{i}\right) \in o_{j \in J_{i}} \Re_{j}$. By Definition [.], for each $i \in I_{\rho(\sigma)}$ we have

$$
\sigma^{\mathfrak{A}}\left(b_{1}^{i-1}, a_{i}^{\rho(\sigma)}\right) \overline{\overline{\Re_{i_{0}} \circ \cdots \circ \Re_{i_{m_{i}}}}} \sigma^{\mathfrak{A}}\left(b_{1}^{i}, a_{i+1}^{\rho(\sigma)}\right)
$$

and by lemma [2.7, we obtain that

$$
\sigma^{\mathfrak{A}}\left(a_{1}^{\rho(\sigma)}\right) \overline{\overline{\Re_{1_{0}} \circ \cdots \circ \Re_{n_{m_{n}}}}} \sigma^{\mathfrak{A}}\left(b_{1}^{\rho(\sigma)}\right) .
$$

Since, $\Re_{1_{0}} \circ \cdots \circ \Re_{n_{m_{n}}} \subseteq \vee_{i \in I} \Re_{i}$ we obtain that

$$
\sigma^{\mathfrak{A}}\left(a_{1}^{\rho(\sigma)}\right) \overline{\overline{\mathrm{V}_{i \in I} \Re_{i}}} \sigma^{\mathfrak{A}}\left(b_{1}^{\rho(\sigma)}\right) .
$$

Thus, $\vee_{i \in I} \Re_{i} \in \operatorname{Reg}_{s}(\mathfrak{A})$ and it implies that $\left(\operatorname{Re}_{s}(\mathfrak{A}), \subseteq\right)$ is a complete sublattice of $(E q(A), \subseteq$ ) .

The regular (resp. strongly regular) lattice of $\mathfrak{A}$ which is denoted by $\boldsymbol{R e g}(\mathfrak{A})\left(\right.$ resp. $\left.\boldsymbol{R e g}_{s}(\mathfrak{A})\right)$, is the lattice whose universe is $\operatorname{Reg}(\mathfrak{A})\left(\operatorname{resp} . \operatorname{Reg}_{s}(\mathfrak{A})\right)$. Let $\mathfrak{A}$ be a $\Sigma$-hyperalgebra. By Theorem [3.3, if $\theta \in E q(\mathfrak{A})$, then by $\operatorname{Reg}(\theta)$ we mean the greatest regular relation which is contained in $\theta$ and clearly we have

$$
\operatorname{Reg}_{\mathfrak{A}}(\theta)=\operatorname{Tr}(\bigcup\{\Re \in \operatorname{Reg}(\mathfrak{A}) \mid \Re \subseteq \theta\}),
$$

and by $\operatorname{Reg}_{s}(\theta)$ we mean the lowest strongly regular relation which contains $\theta$ and we have

$$
\operatorname{Reg}_{s}(\theta)=\bigcap\left\{\Re \in \operatorname{Reg}_{s}(\mathfrak{A}) \mid \theta \subseteq \Re\right\} .
$$

According to [22], if $\mathfrak{A}$ is a $\Sigma$-hyperalgebra then $\boldsymbol{R e g}_{s}(\mathfrak{A})$ is an algebraic closure system so it is an algebraic lattice. By Theorem $\mathbf{3 . 3}$, we can conclude that $(\operatorname{Reg}(\mathfrak{A}), \wedge, \vee, \Delta, \nabla)$ is a bounded lattice.

Proposition 3.4. Let $\mathfrak{A}$ be a $\Sigma$-hyperalgebra. Then $\Delta \in \operatorname{Reg}_{s}(\mathfrak{A})$ if and only if $\mathfrak{A}$ is a $\Sigma$ algebra.

Proof. First let $\Delta \in \operatorname{Reg}_{s}(\mathfrak{A})$. Hence, for each $\sigma \in \Sigma$ and $a_{1}^{\rho(\sigma)} \in A$ we must have $\sigma^{\mathfrak{A}}\left(a_{1}^{\rho(\sigma)}\right) \overline{\bar{\Delta}} \sigma^{\mathfrak{A}}\left(a_{1}^{\rho(\sigma)}\right)$ and it implies that $\left|\sigma^{\mathfrak{A}}\left(a_{1}^{\rho(\sigma)}\right)\right|=1$. Thus $\mathfrak{A}$ is a $\Sigma$-algebra. The converse is trivial.

By Proposition 厍, we obtain that if $\mathfrak{A}$ is not a $\Sigma$-algebra, then $\Delta \notin \operatorname{Reg}_{s}(\mathfrak{A})$ but $\boldsymbol{R e g}_{s}(\mathfrak{A})$ is a complete lattice so it has the lowest bound.

Definition 3.5. Let $\mathfrak{A}$ be a $\Sigma$-hyperalgebra. The lowest bound of complete lattice $\boldsymbol{R e g}_{s}(\mathfrak{A})$ is called the fundamental relation of $\mathfrak{A}$ and it is denoted by $\mathfrak{F}_{\mathfrak{A}}$. 
Theorem 3.6. Let $\mathfrak{A}$ be a $\Sigma$-hyperalgebra and $\Re$ be an equivalence relation of $\mathfrak{A}$. For each $\sigma \in \Sigma$ we define $\sigma^{\mathfrak{A} / \Re}, \dot{\sigma}^{\mathfrak{A} / \Re}:(\mathfrak{A} / \Re)^{\rho(\sigma)} \longrightarrow \mathcal{P}^{*}(\mathfrak{A} / \Re)$, respectively by

$$
\sigma^{\mathfrak{A} / \Re}\left(a_{1}^{\rho(\sigma)} / \Re\right):=\sigma^{\mathfrak{A}}\left(a_{1}^{\rho(\sigma)}\right) / \Re,
$$

and

$$
\dot{\sigma}^{\mathfrak{A} / \Re}\left(a_{1}^{\rho(\sigma)} / \Re\right):=\bigcup_{x_{i} \in a_{i} / \Re}\left(\sigma^{\mathfrak{A}}\left(x_{1}^{\rho(\sigma)}\right) / \Re\right)
$$

Then we have

(1) $\left(A / \Re,\left(\dot{\sigma}^{\mathfrak{A} / \Re}: \sigma \in \Sigma\right)\right)$ is a $\Sigma$-hyperalgebra.

(2) $\Re$ is regular if and only if $\sigma^{\mathfrak{A} / \Re}\left(a_{1}^{\rho(\sigma)} / \Re\right)=\dot{\sigma}^{\mathfrak{A} / \Re}\left(a_{1}^{\rho(\sigma)} / \Re\right)$.

(3) $\Re$ is regular if and only if $\left(A / \Re,\left(\sigma^{\mathfrak{A} / \Re}: \sigma \in \Sigma\right)\right)$ is a $\Sigma$-hyperalgebra.

(4) $\Re$ is strongly regular if and only if $\left(A / \Re,\left(\sigma^{\mathfrak{A} / \Re}: \sigma \in \Sigma\right)\right)$ is a $\Sigma$-algebra.

(5) $\Re$ is strongly regular if and only if $\left(A / \Re,\left(\dot{\sigma}^{\mathfrak{A} / \Re}: \sigma \in \Sigma\right)\right)$ is a $\Sigma$-algebra.

Proof. (1) is proved in [10] and (3) and (4) are proved in [22, 23]. We show only that $\Re$ is regular if and only if $\sigma^{\mathfrak{A} / \Re}$ and $\dot{\sigma}^{\mathfrak{A} / \Re}$ coincide. Clearly, $\sigma^{\mathfrak{A} / \Re}\left(a_{1}^{\rho(\sigma)} / \Re\right) \subseteq \dot{\sigma}^{\mathfrak{A} / \Re}\left(a_{1}^{\rho(\sigma)} / \Re\right)$. Let $\Re$ be regular. Assume that $t / \Re \in \dot{\sigma}^{\mathfrak{A} / \Re}\left(a_{1}^{\rho(\sigma)} / \Re\right)$. So there exist $x_{1}^{\rho(\sigma)}$ such that $x_{i} \in a_{i} / \Re$ and $t / \Re \in \sigma^{\mathfrak{A}}\left(x_{1}^{\rho(\sigma)}\right) / \Re$ and it implies that there is $s \in \sigma^{\mathfrak{A}}\left(x_{1}^{\rho(\sigma)}\right)$ such that $(t, s) \in \Re$. On the other hand, there is $\left.z \in \sigma^{\mathfrak{A}}\left(a_{1}^{\rho(\sigma)}\right)\right)$ such that $(s, z) \in \Re$ and it holds the equality.

Conversely, let $\left(a_{i}, b_{i}\right)_{1}^{\rho(\sigma)} \in \Re$ and $\left.t \in \sigma^{\mathfrak{A}}\left(a_{1}^{\rho(\sigma)}\right) / \Re\right)$. By hypothesis we have

$$
\begin{aligned}
t / \Re \in \sigma^{\mathfrak{A}}\left(a_{1}^{\rho(\sigma)}\right) / \Re & =\sigma^{\mathfrak{A} / \Re}\left(a_{1}^{\rho(\sigma)} / \Re\right)=\dot{\sigma}^{\mathfrak{A} / \Re}\left(a_{1}^{\rho(\sigma)} / \Re\right) \\
& =\bigcup_{x_{i} \in a_{i} / \Re}\left(\sigma^{\mathfrak{A}}\left(x_{1}^{\rho(\sigma)}\right) / \Re\right)=\bigcup_{x_{i} \in b_{i} / \Re}\left(\sigma^{\mathfrak{A}}\left(x_{1}^{\rho(\sigma)}\right) / \Re\right) \\
& =\dot{\sigma}^{\mathfrak{A} / \Re}\left(b_{1}^{\rho(\sigma)} / \Re\right)=\sigma^{\mathfrak{A} / \Re}\left(b_{1}^{\rho(\sigma)} / \Re\right)=\sigma^{\mathfrak{A}}\left(b_{1}^{\rho(\sigma)}\right) / \Re .
\end{aligned}
$$

Thus there is $s \in \sigma^{\mathfrak{A}}\left(b_{1}^{\rho(\sigma)}\right)$ such that $(t, s) \in \Re$. By symmetry we can conclude that $\Re$ is a regular relation. Also, if $\left(A / \Re,\left(\dot{\sigma}^{\mathfrak{A} / \Re}: \sigma \in \Sigma\right)\right)$ is a $\Sigma$-algebra then $\sigma^{\mathfrak{A} / \Re}=\dot{\sigma}^{\mathfrak{A} / \Re}$ and by (4) we obtain (5).

The $\Sigma$-hyperalgebra $\left(A / \Re,\left(\sigma^{\mathfrak{A} / \Re}: \sigma \in \Sigma\right)\right)$ is called the quotient $\Sigma$-hyperalgebra of $\mathfrak{A}$ by $\Re$ and it is denoted by $\mathfrak{A} / \Re$. Also, the $\Sigma$-hyperalgebra $\left(A / \Re,\left(\dot{\sigma}^{\mathfrak{A} / \Re}: \sigma \in \Sigma\right)\right)$ is denoted by $\dot{\mathfrak{A}} / \Re$.

Example 3.7. Consider MV-algebra $\mathfrak{M}$ in Example [2.5. Let

$$
\theta=\{(0,0),(1,1),(a, a),(b, b),(0,1),(a, b),(1,0),(b, a)\}
$$

Then $\dot{\mathfrak{M}} / \theta$ is a hyper MV-algebra of order 2 which is not an MV-algebra. 


\section{Regular RELATions UNDER HOMOMORPHiSMS}

In this section, we study regular and strongly regular relations under homomorphisms. We state and prove the hyperstructure's version of Isomorphism Theorems and Correspondence Theorem. Finally, we show that if $\Re$ is a regular relation which contains $\mathfrak{F}_{\mathfrak{A}}$, then $\Re \in \operatorname{Re} g_{s}(\mathfrak{A})$ and we characterize the fundamental relation of $\mathfrak{A} / \Re$.

Let $f \in \operatorname{Hom}_{G}(\mathfrak{A}, \mathfrak{B})$. Then the kernel relation of $f$, written $\kappa(f)$, is defined by $\kappa(f)=$ $\left\{\left(a, a^{\prime}\right) \in A^{2}: f(a)=f\left(a^{\prime}\right)\right\}$. One can see that $\kappa(f) \in \operatorname{Reg}(\mathfrak{A})$ and if $\mathfrak{B}$ is an algebra, then $\kappa(f) \in \operatorname{Reg}_{s}(\mathfrak{A})$. Let $\theta \in E q(\mathfrak{A})$. The canonical projection $\pi_{\theta}: \mathfrak{A} \longrightarrow \dot{\mathfrak{A}} / \theta$, is defined by $\pi_{\theta}(a)=a / \theta$.

Theorem 4.1. Let $\mathfrak{A}$ be a $\Sigma$-hyperalgebra and $\theta \in E q(\mathfrak{A})$. Then the canonical projection $\pi_{\theta}$ is an onto homomorphism. In particular, $\pi_{\theta}$ is a good homomorphism if and only if $\theta \in \operatorname{Reg}(\mathfrak{A})$.

Proof. Let $\sigma \in \Sigma$ and $a_{1}^{\rho(\sigma)} \in A$. So we have

$$
\begin{aligned}
\pi_{\theta}\left(\sigma^{\mathfrak{A}}\left(a_{1}^{\rho(\sigma)}\right)\right) & =\sigma^{\mathfrak{A}}\left(a_{1}^{\rho(\sigma)}\right) / \theta \\
& \subseteq \bigcup_{x_{i} \in a_{i} / \theta}\left(\sigma^{\mathfrak{A}}\left(x_{1}^{\rho(\sigma)}\right) / \theta\right) \\
& =\dot{\sigma}^{\mathfrak{A} / \theta}\left(\pi_{\theta}\left(a_{1}\right), \cdots, \pi_{\theta}\left(a_{\rho(\sigma)}\right)\right) .
\end{aligned}
$$

Therefore, $\pi_{\theta}$ is a homomorphism. Also, one can see that $\pi_{\theta}$ is an onto map. The second part of theorem is clear by Theorem [3.6.

Theorem 4.2. [110] (Homomorphism Theorem) Let $\mathfrak{A}, \mathfrak{B}$ be $\Sigma$-hyperalgebras and $f: \mathfrak{A} \longrightarrow \mathfrak{B}$ is an onto good homomorphism. Then there is an isomorphism $g: \mathfrak{A} / \operatorname{ker}(f) \longrightarrow \mathfrak{B}$ defined by $f=g \circ \pi_{\kappa(f)}$.

Corollary 4.3. (First isomorphism Theorem) Let $\mathfrak{A}, \mathfrak{B}$ be $\Sigma$-hyperalgebras. $\mathfrak{B}$ is a homomorphic image of $\mathfrak{A}$ if and only if there exists $\Re \in R e g(\mathfrak{A})$ such that $\mathfrak{A} / \Re \cong \mathfrak{B}$. Furthermore, if $\mathfrak{B}$ is an algebra, we can find this $\Re$ in $\operatorname{Reg}_{s}(\mathfrak{A})$.

Now, we describe the behavior of regular and strongly regular relation under homomorphisms and inverse homomorphisms. Let $\mathfrak{A}, \mathfrak{B}$ be two $\Sigma$-hyperalgebras, $f \in \operatorname{Hom}(\mathfrak{A}, \mathfrak{B})$, $\theta \subseteq A^{2}$ and $\eta \subseteq B^{2}$. The image of $\theta$ and the inverse image of $\eta$ are denoted by $f(\theta)$ and $f^{-1}(\theta)$, respectively and defined as follows:

$$
\begin{aligned}
& f(\theta)=\left\{\left(f(a), f\left(a^{\prime}\right)\right) \in B^{2}:\left(a, a^{\prime}\right) \in \theta\right\}, \\
& f^{-1}(\eta)=\left\{\left(a, a^{\prime}\right) \in A^{2}:\left(f(a), f\left(a^{\prime}\right)\right) \in \eta\right\} .
\end{aligned}
$$

Theorem 4.4. Let $\mathfrak{A}, \mathfrak{B}$ be two $\Sigma$-hyperalgebras and $f: \mathfrak{A} \longrightarrow \mathfrak{B}$ be a good homomorphism. Then

(1) for each $\Re \in \operatorname{Reg}(\mathfrak{B}), f^{-1}(\Re) \in \operatorname{Reg}(\mathfrak{A})$, and $\kappa(f) \subseteq f^{-1}(\Re)$. Also, $f^{-1}(\Re) \in \operatorname{Reg}_{s}(\mathfrak{A})$ if and only if $\Re \in \operatorname{Reg}_{s}(\mathfrak{B})$, 
(2) if $f$ is onto then for each $\Re \in \operatorname{Reg}(\mathfrak{A})$, if $\kappa(f) \subseteq \Re$, then $f(\Re) \in \operatorname{Reg}(\mathfrak{B})$. Moreover, $f(\Re) \in \operatorname{Reg}_{s}(\mathfrak{B})$ if and only if $\Re \in \operatorname{Reg}_{s}(\mathfrak{A})$,

(3) for each $\theta \subseteq A^{2}$, if $\kappa(f) \subseteq \operatorname{Reg}_{\mathfrak{A}}(\theta)$, then $f\left(\operatorname{Reg}_{\mathfrak{A}}(\theta)\right)=\operatorname{Reg}_{\mathfrak{B}}(f(\theta))$.

Proof. (1) By Theorem $4 . \mathbb{d}, \pi_{\Re} \circ f: \mathfrak{A} \longrightarrow \mathfrak{B} / \Re$ is a good homomorphism. Also, we have $f^{-1}(\Re)=\kappa\left(\pi_{\Re} o f\right)$, so we get $f^{-1}(\Re) \in \operatorname{Reg}(\mathfrak{A})$. On the other hand, $\kappa(f) \subseteq$ $\kappa\left(\pi_{\Re} O f\right)$ and it completes the proof. Now, assume that $\Re \in \operatorname{Reg}_{s}(\mathfrak{B})$. Since $\mathfrak{B} / \Re$ is a $\Sigma$-algebra, we conclude that $f^{-1}(\Re)=\kappa\left(\pi_{\Re} o f\right) \in \operatorname{Reg}_{s}(\mathfrak{A})$. If $f^{-1}(\Re) \in \operatorname{Reg}_{s}(\mathfrak{A})$, then by Theorem $\mathbf{3 . 6}, \mathfrak{A} / f^{-1}(\Re)$ is a $\Sigma$-algebra and by Theorem 4.2 we obtain that $\mathfrak{A} / f^{-1}(\Re) \cong \mathfrak{B} / \Re$. Therefore, $\mathfrak{B} / \Re$ is a $\Sigma$-algebra and it implies that $\Re \in \operatorname{Reg}_{s}(\mathfrak{B})$.

(2) By the assumption $\kappa(f) \subseteq \Re$, we can show that $f(\Re)$ is an equivalence relation in a routine way. Now, let $\sigma \in \Sigma$ and $\left(b_{i}, b_{i}^{\prime}\right) \in f(\Re)$, for each $i \in I_{\rho(\sigma)}$. Since $f$ is onto, there are $a_{1}^{\rho(\sigma)}, a_{1}^{\prime \rho(\sigma)} \in A$ such that $f\left(a_{i}\right)=b_{i}$ and $f\left(a_{i}^{\prime}\right)=b_{i}^{\prime}$. By hypothesis

$$
\sigma^{\mathfrak{A}}\left(a_{1}^{\rho(\sigma)}\right) \bar{\Re} \sigma^{\mathfrak{A}}\left(a_{1}^{\prime \rho(\sigma)}\right) .
$$

It implies that $f\left(\sigma^{\mathfrak{A}}\left(a_{1}^{\rho(\sigma)}\right)\right) \overline{f(\Re)} f\left(\sigma^{\mathfrak{A}}\left(a_{1}^{\prime \rho(\sigma)}\right)\right)$ and it shows the result. Similarly, we can prove the equality for strongly regular relations. Now, suppose that $f(\Re) \in \operatorname{Reg}_{s}(\mathfrak{B})$. By part (1), we have $f^{-1}(f(\Re)) \in \operatorname{Reg}_{s}(\mathfrak{A})$. Since $\kappa(f) \subseteq \Re$ one can obtain that $f^{-1}(f(\Re))=\Re$ and it states the result.

(3) Let $\Re_{\theta}=\operatorname{Reg}_{\mathfrak{A}}(\theta)$. By hypothesis and part (2) we have $\Re_{\theta} \in \operatorname{Reg}(\mathfrak{B})$. Now, assume that $\Re \in \operatorname{Reg}(\mathfrak{B})$ contains $f(\theta)$. Since $\kappa(f) \subseteq \Re_{\theta}$ one can see that $\theta=f^{-1}(f(\theta)) \subseteq$ $f^{-1}(\Re)$ and by part (1) we have $f^{-1}(\Re) \in \operatorname{Reg}(\mathfrak{A})$. Thus $\Re_{\theta} \subseteq f^{-1}(\Re)$ and it implies that $f\left(\Re_{\theta}\right) \subseteq \Re$. Therefore, $\Re_{\theta}=\operatorname{Reg}_{\mathfrak{B}}(f(\theta))$

Suppose that $\mathfrak{A}$ is a hyperalgebra and $\theta_{1}, \theta_{2} \in E q(\mathfrak{A})$ with $\theta_{1} \subseteq \theta_{2}$. Then let

$$
\theta_{2} / \theta_{1}=\left\{\left(a / \theta_{1}, b / \theta_{1}\right) \in\left(\mathfrak{A} / \theta_{1}\right)^{2}:(a, b) \in \theta_{2}\right\}
$$

Therefore, $\left(a / \theta_{1}, b / \theta_{1}\right) \in \theta_{2} / \theta_{1}$ implies that $(a, b) \in \theta_{2}$.

Theorem 4.5. Let $\mathfrak{A}$ be a $\Sigma$-hyperalgebra and $\theta \in E q(\mathfrak{A})$ and $\mathfrak{S} \in \operatorname{Reg}_{s}(\mathfrak{A})$. Then

(i) $\{\Re / \theta \mid \Re \in \operatorname{Reg}(\mathfrak{A}), \theta \subseteq \Re\} \subseteq \operatorname{Reg}(\dot{\mathfrak{A}} / \theta)$. The equality holds if and only if $\theta \in \operatorname{Reg}(A)$.

(ii) $\left\{\Re / \theta \mid \Re \in \operatorname{Reg}_{s}(\mathfrak{A}), \theta \subseteq \Re\right\} \subseteq \operatorname{Reg}_{s}(\dot{\mathfrak{A}} / \theta)$. If $\theta \in \operatorname{Reg}(\mathfrak{A})$, the equality holds.

(iii) $\operatorname{Con}(\mathfrak{A} / \mathfrak{S})=\{\Re / \mathfrak{S} \mid \Re \in \operatorname{Reg}(\mathfrak{A}), \mathfrak{S} \subseteq \Re\}$.

Proof. (i) The first part is proved in [II]]. Let the equality holds. Then $\Delta_{\dot{\mathfrak{A}} / \theta} \in\{\Re / \theta \mid \Re \in$ $\left.\operatorname{Reg}_{(s)}(\mathfrak{A}), \theta \subseteq \Re\right\}$ and it implies that $\theta \in \operatorname{Reg}(A)$.

(ii) Let $\Re \in \operatorname{Reg}_{s}(\mathfrak{A})$ which is contained $\theta$. Using the good homomorphism $f: \dot{\mathfrak{A}} / \theta \longrightarrow \mathfrak{A} / \Re$, defined by $f(a / \theta)=a / \Re$ and Theorem 4.4 we obtain the inclusion. Also, if $\theta \in \operatorname{Reg}(A)$ we 
can use the projection homomorphism $\pi_{\theta}$ and show the equality.

(iii) Let $\Re \in \operatorname{Reg}(\mathfrak{A})$ and $\mathfrak{S} \subseteq \Re$. Considering the projection $\pi_{\mathfrak{S}}$ we obtain that $\Re / \mathfrak{S} \in$ $\operatorname{Con}(\mathfrak{A} / \mathfrak{S})$. Conversely, if $\eta \in \operatorname{Con}(\mathfrak{A} / \mathfrak{S})$ then $\Re=\pi_{\mathfrak{S}}^{-1}(\eta) \in \operatorname{Reg}_{s}(\mathfrak{A})$ and it implies that $\eta=\Re / \mathfrak{S} \in\{\Re / \mathfrak{S} \mid \Re \in \operatorname{Reg}(\mathfrak{A}), \mathfrak{S} \subseteq \Re\}$.

Example 4.6. Consider hyper MV-algebra $\dot{\mathfrak{M}} / \theta$ in ExAmple [3.7. It is easy to see that $\operatorname{Reg}_{s}(\dot{\mathfrak{M}} / \theta)=\mathfrak{F}_{\mathfrak{\mathfrak { M }} / \theta}=\nabla_{\dot{\mathfrak{M}} / \theta}$. It shows that the equality holds in Theorem 4.5 part $(i i)$.

Theorem 4.7. [110] (Second Isomorphism Theorem) Let $\mathfrak{A}$ be a $\Sigma$-hyperalgebra, $\theta \in E q(\mathfrak{A})$, $\Re \in \operatorname{Reg}(\mathfrak{A})$ and $\theta \subseteq \Re$. Then, there exists an isomorphism from $(\dot{\mathfrak{A}} / \theta) / \Re / \theta$ to $\mathfrak{A} / \Re$.

Let $\mathfrak{A}$ be a $\Sigma$-hyperalgebra and $B \subseteq A$. Then $B$ is called a subhyperuniverse of $\mathfrak{A}$, if for all $\sigma \in \Sigma$ and $b_{1}^{\rho(\sigma)} \in B$ we have $\sigma^{\mathfrak{A}}\left(b_{1}^{\rho(\sigma)}\right) \subseteq B$. The set of all subhyperuniverses of $\mathfrak{A}$ will be denoted by $S u b(\mathfrak{A})$. Suppose that $B$ is a subset of $A$ and $\Re$ is a regular relation on $\mathfrak{A}$. According to [2:1], $S u b(\mathfrak{A})$ is an algebraic closed set system and the closure operator associated with this closed set system is denoted by $S g^{\mathfrak{A}}$. Also, if $\mathfrak{B}$ is a $\Sigma$-hyperalgebra such that $B \in S u b(\mathfrak{A})$ we say that $\mathfrak{B}$ is a subhyperalgebra of $\mathfrak{A}$ and we write $\mathfrak{B} \leq \mathfrak{A}$. Let $B^{\Re}=\{a \in A: B \cap a / \Re \neq \emptyset\}$. Let $\mathfrak{B}^{\Re}=S g^{\mathfrak{A}}\left(B^{\Re}\right)$. Also, we define $\left.\Re\right|_{B}=\Re \cap B^{2}$ and call it the restriction of $\Re$ to $B$.

Lemma 4.8. Let $\mathfrak{A}$ be a $\Sigma$-hyperalgebra and $\mathfrak{B} \leq \mathfrak{A}$. Then

(1) the universe of $\mathfrak{B}^{\Re}$ is $B^{\Re}$.

(2) if $\Re \in \operatorname{Reg}(\mathfrak{A})$, then $\left.\Re\right|_{B} \in \operatorname{Reg}(\mathfrak{B})$.

(3) if $\Re \in \operatorname{Reg}_{s}(\mathfrak{A})$, then $\left.\Re\right|_{B} \in \operatorname{Reg}_{s}(\mathfrak{B})$.

Proof. Let $\sigma \in \Sigma$ and $a_{1}^{\rho(\sigma)} \in B^{\Re}$. So we can find $b_{1}^{\rho(\sigma)} \in B$ such that $(a, b)_{1}^{\rho(\sigma)} \in \Re$, for each $i \in I_{\rho(\sigma)}$. Now. we have

$$
\sigma^{\mathfrak{A}}\left(a_{1}^{\rho(\sigma)}\right) \bar{\Re} \sigma^{\mathfrak{A}}\left(b_{1}^{\rho(\sigma)}\right)
$$

and it implies that for each $u \in \sigma^{\mathfrak{A}}\left(a_{1}^{\rho(\sigma)}\right)$ there is a $v \in \sigma^{\mathfrak{A}}\left(b_{1}^{\rho(\sigma)}\right)$ such that $u \Re v$ so $u \in B^{\Re}$. Thus $B^{\Re}$ is a subuniverse of $\mathfrak{A}$. Next, to verify $\left.\Re\right|_{B} \in \operatorname{Reg}(\mathfrak{B})$ is straightforward.

Theorem 4.9. (Third Isomorphism Theorem) Let $\mathfrak{A}$ be a $\Sigma$-hyperalgebra, $\mathfrak{B} \leq \mathfrak{A}$ and $\Re \in$ $\operatorname{Reg}(\mathfrak{A})$. Then

$$
\mathfrak{B} /\left.\Re\right|_{B} \cong \mathfrak{B}^{\Re} /\left.\Re\right|_{B^{\Re}}
$$

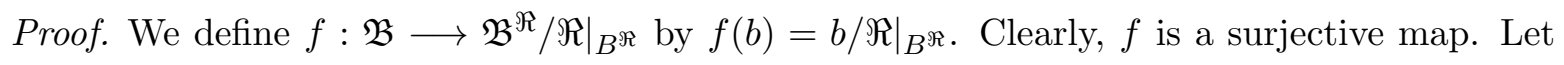
$\sigma \in \Sigma$ and $b_{1}^{\rho(\sigma)} \in B$. Clearly,

$$
f\left(\sigma^{\mathfrak{B}}\left(b_{1}^{\rho(\sigma)}\right)\right) \subseteq \sigma^{\mathfrak{B}^{\Re} /\left.\Re\right|_{B^{\Re}}}\left(f\left(b_{1}\right), \cdots, f\left(b_{\rho(\sigma)}\right)\right) .
$$

Let $u /\left.\Re\right|_{B^{\Re}} \in \sigma^{\mathfrak{B}^{\Re} /\left.\Re\right|_{B^{\Re}}}\left(f\left(b_{1}\right), \cdots, f\left(b_{\rho(\sigma)}\right)\right)$. So there exists $v \in \sigma^{\mathfrak{B}^{\Re}}\left(b_{1}^{\rho(\sigma)}\right)$ such that $u /\left.\Re\right|_{B^{\Re}}=v /\left.\Re\right|_{B^{\Re}}$. By Lemma $4.8, v \in \sigma^{\mathfrak{B}}\left(b_{1}^{\rho(\sigma)}\right)$, so $f(v)=v /\left.\Re\right|_{B^{\Re}}$ and it implies that 
$f$ is a good homomorphism. Also, $\kappa(f)=\left.\Re\right|_{B}$. Therefore, by Theorem 4.2, we can get the result.

The Correspondence Theorem in this section plays an important role in the study of $\Sigma$ hyperalgebras. This theorem shows that the regular and strongly regular lattice of every homomorphic image of a $\Sigma$-hyperalgebra is isomorphically embeddable as a special kind of sublattice of the regular lattice of a $\Sigma$-hyperalgebra.

Theorem 4.10. (Correspondence Theorem). Let $\mathfrak{A}$ be a $\Sigma$-hyperalgebra, $\Re \in \boldsymbol{B}(\operatorname{Reg}(\mathfrak{A}))$ and $\mathfrak{S} \in \operatorname{Reg}_{s}(\mathfrak{A})$. Then

(1) $\left[\Re, \nabla_{\mathfrak{A}}\right]_{\operatorname{Reg}(\mathfrak{A})}$ is isomorphic to $\boldsymbol{R e g}(\mathfrak{A} / \Re)$ as a lattice.

(2) $\left[\mathfrak{S}, \nabla_{\mathfrak{A}}\right]_{\operatorname{Reg}(\mathfrak{A})}$ is isomorphic to $\operatorname{Con}(\mathfrak{A} / \mathfrak{S})$ as a lattice.

(3) $\left[\mathfrak{S}, \nabla_{\mathfrak{A}}\right]_{\operatorname{Reg}_{s}(\mathfrak{A})}$ is isomorphic to $\operatorname{Con}(\mathfrak{A} / \mathfrak{S})$ as a lattice.

Proof. $\quad$ (1) We define the mapping

$$
\varphi:\left[\Re, \nabla_{\mathfrak{A}}\right]_{\operatorname{Reg}(\mathfrak{A})} \longrightarrow \boldsymbol{\operatorname { R e g }}(\mathfrak{A} / \Re)
$$

by $\varphi\left(\Re^{\prime}\right)=\pi_{\Re}\left(\Re^{\prime}\right)$, for each $\Re^{\prime} \in\left[\Re, \nabla_{\mathfrak{A}}\right]_{\operatorname{Reg}(\mathfrak{A})}$. By Theorem 4.4 , $\varphi$ is a welldefined map. Let $\Re_{1}, \Re_{2} \in\left[\Re, \nabla_{\mathfrak{A}}\right]_{R e g(\mathfrak{A})}$. Let $\pi_{\Re}\left(\Re_{1}\right)=\pi_{\Re}\left(\Re_{2}\right)$ and $(x, y) \in \Re_{1}$. So $(x / \Re, y / \Re) \in \pi_{\Re}\left(\Re_{1}\right)=\pi_{\Re}\left(\Re_{2}\right)$ and it implies that $(x, y) \in \Re_{2}$. It states that $\Re_{1} \subseteq \Re_{2}$. By symmetry, we obtain that $\Re_{2} \subseteq \Re_{1}$, too. It shows that $\varphi$ is one-to-one. Now, assume that $\mathfrak{T} \in \operatorname{Reg}(\mathfrak{A} / \Re)$. By Theorem $4.4, \pi^{-1}(\mathfrak{T}) \in \operatorname{Reg}(\mathfrak{A})$ which contains $\Re$. One can see that $\varphi\left(\pi^{-1}(\mathfrak{T})\right)=\mathfrak{T}$. Therefore, $\varphi$ is a one-to-one correspondence. Finally, it is easy to see that $\varphi$ is monotone and it states that $\varphi$ is an isomorphism. Similarly, we can prove (2) and (3).

In Theorem 4.1], we show that each regular relation which contains a strongly regular relation is a strongly regular relation, too. See Figure $\mathbb{\square}$.

Theorem 4.11. Let $\mathfrak{A}$ be a $\Sigma$-hyperalgebra and $\Re \in R e g(\mathfrak{A})$ such that $\mathfrak{F}_{\mathfrak{A}} \subseteq \Re$. Then $\Re \in$ $\operatorname{Reg}_{s}(\mathfrak{A})$.

Proof. By Theorem $4.10, \pi_{\mathfrak{F}_{\mathfrak{A}}}(\Re) \in \operatorname{Con}\left(\mathfrak{A} / \mathfrak{F}_{\mathfrak{A}}\right)$. On the other hand, $\operatorname{Con}\left(\mathfrak{A} / \mathfrak{F}_{\mathfrak{A}}\right) \cong$ $\left[\mathfrak{F}_{\mathfrak{A}}, \nabla_{\mathfrak{A}}\right]_{\operatorname{Reg}_{s}(\mathfrak{A})}$ so there exists $\mathfrak{S} \in\left[\mathfrak{F}_{\mathfrak{A}}, \nabla_{\mathfrak{A}}\right]_{\operatorname{Reg}_{s}(\mathfrak{A})}$ such that $\pi_{\mathfrak{F}_{\mathfrak{A}}}(\mathfrak{S})=\pi_{\mathfrak{F}_{\mathfrak{A}}}(\Re)$ and it implies that $\mathfrak{S}=\Re$. Hence, $\Re \in \operatorname{Reg}_{s}(\mathfrak{A})$.

Corollary 4.12. Let $\mathfrak{A}$ be a $\Sigma$-hyperalgebra and $\Re \in \boldsymbol{B}(\operatorname{Reg}(\mathfrak{A}))$. Then $\mathfrak{F}_{\mathfrak{A} / \Re}=\left(\mathfrak{F}_{\mathfrak{A}} \vee \Re\right) / \Re$. In particular, if $\Re \subseteq \mathfrak{F}_{\mathfrak{A}}$ then $\mathfrak{F}_{\mathfrak{A} / \Re}=\mathfrak{F}_{\mathfrak{A}} / \Re$. 


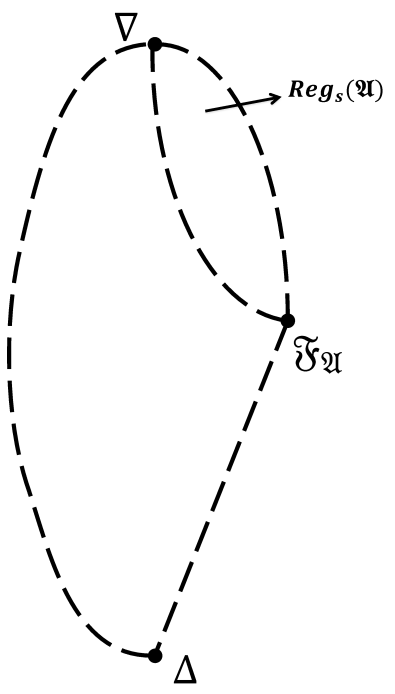

Figure 1. The lattice of regular relations of $\mathfrak{A}$.

Proof. Consider the projection $\pi_{\Re}$. By Theorem 4.4 , we have $\pi^{-1}\left(\mathfrak{F}_{\mathfrak{A} / \Re}\right) \in \operatorname{Reg}(\mathfrak{A})$ which

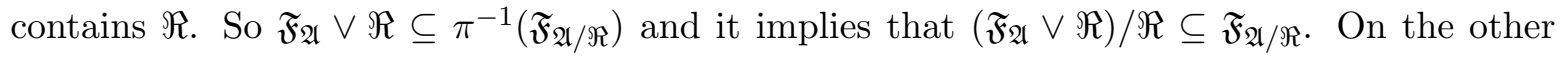
hand by Theorem 4.5 and Lemma $4 . \Pi,\left(\mathfrak{F}_{\mathfrak{A}} \vee \Re\right) / \Re \in R e g_{s}(\mathfrak{A} / \Re)$ so we have $\mathfrak{F}_{\mathfrak{A} / \Re} \subseteq\left(\mathfrak{F}_{\mathfrak{A}} \vee \Re\right) / \Re$ and it proves the theorem.

In the following, we want to study the structure of the fundamental relation for an arbitrary $\Sigma$-hyperalgebra. This relation has an important role in the study of $\Sigma$-hyperalgebras. Let $\mathfrak{A}$ be a $\Sigma$-hyperalgebra and $X \subseteq A$. We define

$$
F(X)=\left\{\sigma^{\mathfrak{A}}\left(a_{1}^{\rho(\sigma)}\right): \forall \sigma \in \Sigma, a_{1}^{\rho(\sigma)} \in X\right\}
$$

Now, we define $F^{n}(X)$ for $n \geq 1$ by

$$
\begin{aligned}
& F^{1}(X)=F(X), \\
& F^{n+1}(X)=F\left(F^{n}(X) \cup X\right) .
\end{aligned}
$$

Clearly, $F^{1}(X) \subseteq F^{2}(X) \subseteq \cdots$. Let $\mathcal{C}(X)=\cup_{i=1}^{\infty} F^{i}(X)$

Definition 4.13. Let $\mathfrak{A}$ be a $\Sigma$-hyperalgebra. We define a binary relation $\mathfrak{f}_{\mathfrak{A}}$ on $A$ as follows.

$$
(x, y) \in f_{\mathfrak{A}} \text { if and only if } \exists X \subseteq_{w} A, z \in \mathcal{C}(X)(\{x, y\} \subseteq z) .
$$

Let $\mathfrak{f}_{\mathfrak{A}}=E q\left(f_{\mathfrak{A}}\right)$. Since, $f_{\mathfrak{A}}$ is symmetric we have $\mathfrak{f}_{\mathfrak{A}}=\operatorname{Tr}\left(f_{\mathfrak{A}}\right) \cup \Delta$.

Lemma 4.14. Let $\mathfrak{A}$ be a $\Sigma$-hyperalgebra and $\Re \in \operatorname{Reg}_{s}(\mathfrak{A})$. Then $f_{\mathfrak{A}} \subseteq \Re$. In particular, $\mathfrak{f}_{\mathfrak{A}} \subseteq \Re$. 
Proof. By Theorem [3.6, $\mathfrak{A} / \Re$ is a $\Sigma$-algebra. Consider the canonical projection $\pi_{\Re}: \mathfrak{A} \longrightarrow$ $\mathfrak{A} / \Re$. Let $(a, b) \in f_{\mathfrak{A}}$, so there exist $X \subseteq_{w} A$ and $z \in \mathcal{C}(X)$ such that $\{x, y\} \subseteq z$. Now, we have $\pi_{\Re}(a), \pi_{\Re}(b) \subseteq \pi_{\Re}(z)$ but $\left|\pi_{\Re}(z)\right|=1$ and it implies $\pi_{\Re}(a)=\pi_{\Re}(b)$ so $(a, b) \in \Re$. Since, $\Re$ is an equivalence relation we have $\mathfrak{f}_{\mathfrak{A}} \subseteq \Re$.

Theorem 4.15. $f_{\mathfrak{A}}$ has the strongly regular substitution property on $\Sigma$-hyperalgebra $\mathfrak{A}$.

Proof. Let $\sigma \in \Sigma$ and $(a, b)_{1}^{\rho(\sigma)} \in f_{\mathfrak{A}}$. So there exists $X_{i} \subseteq_{w} A$ and $z_{i} \in \mathcal{C}\left(X_{i}\right)$ such that $\left\{a_{i}, b_{i}\right\} \subseteq z_{i}$. Now, for each $i$ we have $\sigma^{\mathfrak{A}}\left(b_{1}^{i-1}, a_{i}^{\rho(\sigma)}\right)$ and $\sigma^{\mathfrak{A}}\left(b_{1}^{i}, a_{i+1}^{\rho(\sigma)}\right)$ are subsets of $\sigma^{\mathfrak{A}}\left(b_{1}^{i-1}, z_{i}, a_{i+1}^{\rho(\sigma)}\right)$. Now, if we consider $a \in \sigma^{\mathfrak{A}}\left(a_{1}^{\rho(\sigma)}\right)$ and $b \in \sigma^{\mathfrak{A}}\left(b_{1}^{\rho(\sigma)}\right)$ then $\{a, b\} \subseteq$ $\sigma^{\mathfrak{A}}\left(z_{1}^{\rho(\sigma)}\right) \in \mathcal{C}(X)$, where $X \subseteq \cup_{i=1}^{\rho(\sigma)} X_{i}$.

Corollary 4.16. $\mathfrak{f}_{\mathfrak{A}}$ is the smallest strongly regular relation on $A$, i. e. $\mathfrak{F}_{\mathfrak{A}}=\mathfrak{f}_{\mathfrak{A}}$.

Proof. By Theorem 4.55, $f_{\mathfrak{A}}$ has the strongly regular substitution property and by Lemma [.9, we conclude that $\operatorname{Tr}\left(f_{\mathfrak{A}}\right)$ and consequently $\mathfrak{f}_{\mathfrak{A}}$ have the strongly regular substitution property, too. So $\mathfrak{f}_{\mathfrak{A}}$ is a strongly regular relation on $A$. On the other hand, by Lemma $4.14, \mathfrak{f}_{\mathfrak{A}}$ is the subset of each strongly regular relation. It holds the result.

According to Corollary $4.16, \mathfrak{f}_{\mathfrak{A}}$ is the fundamental relation $\mathfrak{F}_{\mathfrak{A}}$ on $A$. The $\Sigma$-algebra $\mathfrak{A} / \mathfrak{F}_{\mathfrak{A}}$ is called the fundamental $\Sigma$-algebra.

\section{Complete parts}

Complete parts were introduced and studied by M. Koskas in [15]. Later, this topic was analyzed by P. Corsini in [2] and Y. Sureau in [3] mostly in the general theory of hypergroups. Complete parts and fundamental relations are very closely related. In fact, the equivalence relations of a fundamental relation form a complete part.

Definition 5.1. Let $\mathfrak{A}$ be a $\Sigma$-hyperalgebra and $B$ be a nonempty subset of $A$. We say that $B$ is a complete part of $A$ if for each $\sigma \in \Sigma$ and $X \subseteq_{w} A$, the following implication holds:

$$
\forall z \in \mathcal{C}(X)(B \cap z \neq \emptyset \Longrightarrow z \subseteq B)
$$

The set of all complete parts of $\mathfrak{A}$ with $\emptyset$ is denoted by $\operatorname{Comp}(\mathfrak{A})$.

Proposition 5.2. Let $\mathfrak{A}$ be a $\Sigma$-hyperalgebra and $\Re \in R e g(\mathfrak{A})$. Then $\Re$ is strongly regular if and only if for each $x \in A, x / \Re \in \operatorname{Comp}(\mathfrak{A})$.

Proof. First let $\Re$ be a strongly regular relation. Suppose that $X \subseteq_{w} A$ and $x \in A$ such that $C \cap x / \Re \neq \emptyset$, for some $C \in \mathcal{C}(X)$. So there exists $y \in C$ such that $y \Re x$. Assume that $z \in C$. Thus $y \mathfrak{f}_{\mathfrak{A}} z$ so by Theorem 4.16 we obtain that $y \Re z$ and it implies that $z \in x / \Re$. 
Conversely, let $\Re \in \operatorname{Reg}(\mathfrak{A})$ and $x / \Re$ is a complete part for each $x \in A$. Let $\sigma \in \Sigma$ and $x_{1}^{\rho(\sigma)}, y_{1}^{\rho(\sigma)} \in A$ such that $x_{i} \Re y_{i}$, for all $i \in\{1, \cdots, \rho(\sigma)\}$. Since $\Re$ is regular we get that $\sigma^{\mathfrak{A}}\left(x_{1}^{\rho(\sigma)}\right) \bar{\Re} \sigma^{\mathfrak{A}}\left(y_{1}^{\rho(\sigma)}\right)$. Now, assume that $x \in \sigma^{\mathfrak{A}}\left(x_{1}^{\rho(\sigma)}\right)$ so there exists $y \in \sigma^{\mathfrak{A}}\left(y_{1}^{\rho(\sigma)}\right)$ such that $x \Re y$ and by hypothesis we conclude that $\sigma^{\mathfrak{A}}\left(y_{1}^{\rho(\sigma)}\right) \subseteq x / \Re$. It implies that $\Re \in \operatorname{Reg}_{s}(\mathfrak{A})$.

Theorem 5.3. Let $\mathfrak{A}$ be a $\Sigma$-hyperalgebra. Then $\mathfrak{C o m p}(\mathfrak{A})=(\operatorname{Comp}(\mathfrak{A}), \cap, \cup, \emptyset, A)$ is a boolean algebra.

Proof. Let $\left\{B_{i}\right\}_{i \in I}$ be a nonempty family of complete parts of $\mathfrak{A}$. Suppose that $X \subseteq_{w} A$ such that $C \cap\left(\cap_{i \in I} B_{i}\right) \neq \emptyset$, for some $C \in \mathcal{C}(X)$. So for each $i \in I$ we have $C \cap B_{i} \neq \emptyset$. Since $B_{i} \in \operatorname{Comp}(\mathfrak{A})$ we obtain that $C \subseteq \cap_{i \in I} B_{i}$. Similarly, one can check that $\cup_{i \in I} B_{i} \in \operatorname{Comp}(\mathfrak{A})$. Now, let $B$ be a complete part. Suppose that $X \subseteq_{w} A$ such that $C \cap B^{c} \neq \emptyset$, for some $C \in \mathcal{C}(X)$. Obviously, $C \cap B=\emptyset$ and it implies that $B^{c} \in C \operatorname{Comp}(\mathfrak{A})$. Therefore $\operatorname{Comp}(\mathfrak{A})$ is a boolean algebra.

Let $C: \mathcal{P}(A) \longrightarrow \mathcal{P}(A)$ be the closure operator associated with boolean algebra $\mathfrak{C o m p}(\mathfrak{A})$. Clearly, for each $X \subseteq A$ we have $C(X)=\cap\{C \mid X \subseteq C \in C o m p(\mathfrak{A})\}$.

Proposition 5.4. Let $\mathfrak{A}$ be a $\Sigma$-hyperalgebra and $C: \mathcal{P}(A) \longrightarrow \mathcal{P}(A)$ be the closure operator associated with boolean algebra $\mathfrak{C o m p}(\mathfrak{A})$. Then for each $X, Y \subseteq A$ and family of subsets of $A$, $\left\{X_{i}\right\}_{i \in I}$, we have

1) $X \subseteq C(X)$,

2) $C(C(X))=C(X)$

3) $X \subseteq Y$ implies that $C(X) \subseteq C(Y)$,

4) $C(A)=A$,

5) $C\left(\cup_{i \in I} X_{i}\right)=\cup_{i \in I} C\left(X_{i}\right)$. In particular, $C(X)=\cup_{x \in X} C(x)$,

6) $C\left(\cap_{i \in I} X_{i}\right) \subseteq \cap_{i \in I} C\left(X_{i}\right)$, and equality holds if each $X_{i}$ is a complete part.

Proof. By closure operator properties (1), (2) and (3) are obvious. Also, by part (1), we have $A \subseteq C(A)$ and it implies $C(A)=A$.

(5) By part (3), we conclude that $\cup_{i \in I} C\left(X_{i}\right) \subseteq C\left(\cup_{i \in I} X_{i}\right)$. On the other hand, by part (1), we obtain that $\cup_{i \in I} X_{i} \subseteq \cup_{i \in I} C\left(X_{i}\right)$. So by part (3), $C\left(\cup_{i \in I} X_{i}\right) \subseteq C\left(\cup_{i \in I} C\left(X_{i}\right)\right)$. By Theorem 5.31, $\cup_{i \in I} C\left(X_{i}\right) \in \operatorname{Comp}(\mathfrak{A})$ and it holds the result.

(6) By part (3) we obtain that $C\left(\cap_{i \in I} X_{i}\right) \subseteq \cap_{i \in I} C\left(X_{i}\right)$. Clearly, if each $X_{i}$ is a complete part than the equality holds.

In the following, for a subset $X$ of the $\Sigma$-hyperalgebra $A$ we denote $K_{1}(X)=X$ and for all $n \geq 1$ we denote

$$
K_{n+1}(X)=\left\{x \in A \mid \exists Y \subseteq w A, \exists z \in \mathcal{C}(Y)\left(x \in z \wedge z \cap K_{n}(X) \neq \emptyset\right)\right\}
$$


Let $K(X)=\cup_{n \in \mathbb{N}} K_{n}(X)$. Obviously, $K(\emptyset)=\emptyset$ and $X \subseteq Y$ implies that $K_{n}(X) \subseteq K_{n}(Y)$, for each integers $n$.

Theorem 5.5. Let $\mathfrak{A}$ be a $\Sigma$-hyperalgebra and $X$ be a nonempty subset of $A$. Then $C(X)=$ $K(X)$.

Proof. First, we show that $K(X)$ is a complete part. Suppose that there exists finite subset $Y$ of $A$ such that $z \cap K(X) \neq \emptyset$, for some $z \in \mathcal{C}(Y)$. So there is an integer $n$ such that $z \cap K_{n}(X) \neq \emptyset$ and it implies that $z \subseteq K_{n+1}(X)$ and it proves that $K(X) \in \operatorname{Comp}(\mathfrak{A})$.

Now, assume that $C \in \operatorname{Comp}(\mathfrak{A})$ such that $X \subseteq C$. By induction we show that $K_{n}(X) \subseteq C$, for each integer $n$. Clearly, $K_{1}(X) \subseteq C$. Now, let $K_{i}(X) \subseteq C$, for some integer $i$ and $x \in K_{i+1}(X)$. So there exists a finite subset $Y$ of $A$ such that $z \cap K_{i}(X) \neq \emptyset$, for some $z \in \mathcal{C}(Y)$. By hypothesis we obtain that $z \subseteq C$ and it shows that $K_{i+1}(X) \subseteq C$. Hence, $K(X) \subseteq C$ and it implies that $C(X)=K(X)$.

Theorem 5.6. Let $\mathfrak{A}$ be a $\Sigma$-hyperalgebra and $x, y \in A$. Then

(i) For all $n \geq 2$ we have $K_{n}\left(K_{2}(x)\right)=K_{n+1}(x)$.

(ii) the next equivalence holds for $n \geq 2$ :

$$
x \in K_{n}(y) \Longleftrightarrow y \in K_{n}(x) .
$$

Proof. (i) Clearly, $K_{1}\left(K_{2}(x)\right)=K_{2}(x)$. Now, assume that $K_{i}\left(K_{2}(x)\right)=K_{i+1}(x)$. We have

$$
K_{i+1}\left(K_{2}(x)\right)=\left\{a \in A \mid \exists Z \subseteq_{w} A, \exists z \in \mathcal{C}(Z)\left(a \in z \wedge z \cap K_{i}\left(K_{2}(x)\right) \neq \emptyset\right)\right\}
$$

and it shows that $K_{i+1}\left(K_{2}(x)\right)=K_{i+2}(x)$. It holds the result.

(ii) We check the equivalence by induction. Let $n=2$. By $x \in K_{2}(y)$ we conclude that there is a finite subset $Z$ of $A$ such that $x \in z$ and $z \cap K_{1}(y) \neq \emptyset$, for some $z \in \mathcal{C}(Z)$. It implies that $\{x, y\} \subseteq z$ so $y \in K_{2}(x)$. Now, let the equivalence holds for $n=i$. Assume that $x \in K_{i+1}(y)$ so there is a finite subset $Z$ of $A$ such that $x \in z$ and $z \cap K_{i}(y) \neq \emptyset$, for some $z \in \mathcal{C}(Z)$. Let $v \in z \cap K_{i}(y) \neq \emptyset$. It follows that $v \in K_{2}(x)$ and $y \in k_{i}(v)$. Thus by part (i), we have $y \in K_{i}\left(K_{2}(x)\right)=K_{i+1}(x)$. Thus the equivalence holds.

Corollary 5.7. Let $\mathfrak{A}$ be a $\Sigma$-hyperalgebra. The binary relation defined as follows:

$$
\forall x, y \in A,(x, y) \in \mathfrak{K} \Longleftrightarrow \exists n \in \mathbb{N}, x \in K_{n}(y)
$$

is an equivalence relation and for each $x \in A, x / \mathfrak{K}=C(x)$.

Let $W_{A} \subseteq A$ be the diagonal set of equivalence classes of the relation $\mathfrak{K}$.

Corollary 5.8. Let $\mathfrak{A}$ be a $\Sigma$-hyperalgebra and $X \subseteq A$. Then

(i) $C(\emptyset)=\emptyset$ if and only if $\operatorname{Comp}(\mathfrak{A}) \neq\{\emptyset, A\}$, 
(ii) $C\left(W_{A}\right)=A$,

(iii) if $X \subseteq A$, then $C(X)$ is the union of disjoint complete parts of $A$.

(iv) $\operatorname{Comp}(\mathfrak{A})$ is an atomic boolean algebra which means that every non-zero element of $\mathfrak{C o m p}(\mathfrak{A})$ contains an atom.

Proof. (i) Clearly, $C(\emptyset)=\cap\{C \mid C$ is complete part $\}$. Let $C \neq A$ be a complete part of $\mathfrak{A}$. Thus by Theorem 5.3, $C^{c} \in \operatorname{Comp}(\mathfrak{A})$. Let $x \in C$ and $y \in C^{c}$. Assume that $C(x) \cap C(y) \neq \emptyset$. By Corollary [5.7, $y \in C(x) \subseteq C$ and it is a contradiction. Hence, $C(x) \cap C(y)=\emptyset$ and it implies that $C(\emptyset)=\emptyset$. Conversely, if $\operatorname{Comp}(\mathfrak{A})=\{\emptyset, A\}$, then $C(\emptyset)=A$. It proves the corollary.

(ii) By Proposition 5.4 and Corollary 5.7, it is obvious.

(iii) Let $x \in W_{A}$ such that $C(x) \cap X \neq \emptyset$. Suppose that $y \in C(x) \cap X$ so by Corollary 5.7 and Proposition 5.4, $C(x)=C(y) \subseteq C(X)$. Therefore, $\cup\left\{C(x) \mid x \in W_{A}, C(x) \cap X \neq \emptyset\right\} \subseteq C(X)$. Now, assume that $a \in C(X)$. By Proposition 5.4, there is $x \in X$ such that $a \in C(x)$. On the other hand, $x \in C\left(W_{A}\right)$. Thus, there exists $y \in W_{A}$ such that $x \in C(y)$ and it implies that $a \in C(y)$. It satisfies the equality.

(iv) By Theorem 5.3, $\mathfrak{C o m p}(\mathfrak{A})$ is a boolean algebra. Also, It is obvious that $C(x)$ is an atom, for each $x \in W_{A}$ and by Proposition 5.4, $\bigcup_{x \in W_{A}} C(x)=C\left(W_{A}\right)=A$. Hence, the unit element of $\mathfrak{C o m p}(\mathfrak{A})$ is the sum of all the atoms that it contains. Thus according to Theorem 5.3 of [9], $\mathfrak{C o m p}(\mathfrak{A})$ is an atomic boolean algebra.

Lemma 5.9. Let $\mathfrak{A}$ be a $\Sigma$-hyperalgebra and $x, y \in A$. Then for integers $n \geq 2$ we have $y \in K_{n}(x)$ if and only if $(x, y) \in f_{\mathfrak{A}}^{n-1}$.

Proof. We prove this lemma by induction on $n$. Clearly, $y \in K_{2}(x)$ if and only if $(x, y) \in f_{\mathfrak{A}}$. Let

$$
y \in K_{n-1}(x) \Longleftrightarrow(x, y) \in f_{\mathfrak{A}}^{n-2} .
$$

Now, assume that $x \in K_{n}(y)$ which means that there is a finite subset $Z$ of $A$ and $z \in \mathcal{C}(Z)$ such that $x \in z$ and $z \cap K_{n-1}(y) \neq \emptyset$. Let $u \in z \cap K_{n-1}(y)$, so $(x, u) \in f_{\mathfrak{A}}$ and $(u, y) \in f_{\mathfrak{A}}^{n-2}$ which states $(x, y) \in f_{\mathfrak{A}}^{n-1}$. Conversely, suppose that $(x, y) \in f_{\mathfrak{A}}^{n-1}$. So there is $u \in A$ such that $(x, u) \in f_{\mathfrak{A}}$ and $(u, y) \in f_{\mathfrak{A}}^{n-2}$. Hence, we conclude that $u \in K_{2}(x)$ and $y \in K_{n-1}(u)$. Therefore, by Theorem 5.6, $y \in K_{n-1}(u) \subseteq K_{n-1}\left(K_{2}(x)\right)=K_{n}(x)$. It holds the result.

Theorem 5.10. Let $\mathfrak{A}$ be a $\Sigma$-hyperalgebra. The equivalence relations $\mathfrak{K}$ and $\mathfrak{f}_{\mathfrak{A}}$ coincide.

Proof. Let $(x, y) \in f_{\mathfrak{A}}$. There is a finite subset $Z$ of $A$ and $z \in \mathcal{C}(Z)$ such that $\{x, y\} \subseteq z$. So $x \in K_{2}(y)$ and it implies that $(x, y) \in \mathfrak{K}$. Hence, $f_{\mathfrak{A}} \subseteq \mathfrak{K}$, whence $\mathfrak{f}_{\mathfrak{A}} \subseteq \mathfrak{K}$. Conversely, let $(x, y) \in \mathfrak{K}$. So there exists an integer $n$ such that $y \in K_{n}(x)$. By Lemma 5.9, we obtain that $(x, y) \in f_{\mathfrak{A}}^{n-1} \subseteq \mathfrak{f}_{\mathfrak{A}}$. 
Theorem 5.11. Let $\mathfrak{A}$ be a $\Sigma$-hyperalgebra. Then $f_{\mathfrak{A}}$ is transitive if and only if for all $x \in W_{A}$ we have $C(x)=K_{2}(x)$.

Proof. First, let for all $x \in W_{A}, C(x)=K_{2}(x)$. We show that $f_{\mathfrak{A}}=\mathfrak{K}$. By Theorem 5.10, we have $f_{\mathfrak{A}} \subseteq \mathfrak{K}$. Now, let $(x, y) \in \mathfrak{K}$. So there exists an integer $n$ such that $x \in K_{n}(y) \subseteq K(y)=$ $C(y)$. Also, there is a $u \in W_{A}$ such that $y \in C(u)=K_{2}(u)$ and by Theorem $5 \mathbf{5 6}$, we obtain that $C(y)=K_{2}(y)$. It states that $x \in K_{2}(y)$ so $(x, y) \in f_{\mathfrak{A}}$. Conversely, let $f_{\mathfrak{A}}$ is transitive. Assume that $y \in C(x)=K(x)$, for some $x \in W_{A}$. So there exists an integer $n$ such that $y \in K_{n}(x)$. By Lemma 5.9, we have $(x, y) \in f_{\mathfrak{A}}^{n-1}=f_{\mathfrak{A}}$ and it implies $y \in K_{2}(x)$.

\section{Conclusion}

The above discussion shows that we can extend some notions of hypergroup theory to a $\Sigma$-hyperalgebra for an arbitrary signature $\Sigma$. This paper provides suitable tools for doing more research in the area of hyperstructures, such as on fundamental relations and quotient hyperalgebras.

\section{REFERENCES}

[1] G. Birkhoff, Lattice Theory, 3rd edition. Colloq. Publ., vol. 25, Amer. Math. Soc., Providence, (1967).

[2] P. Corsini, Contributo alla teoria degli ipergruppi, Atti Soc. Pelor. Sc. Mat. Fis. Nat. Messina, Messina, Italy, (1980), pp. 1-22.

[3] P. Corsini and V. Leoreanu, Applications of Hyperstructure Theory, Kluwer, Dordrecht, (2003).

[4] B. Davvaz and V. Leoreanu-Fotea, Hyperring Theory and Applications, International Academic Press, USA, (2007).

[5] B. Davvaz and T. Vougiouklis, n-ary hypergroups, Iran. J. Sci. Technol. Trans. A Sci., 30(2) (2006), $165-174$.

[6] B. Davvaz and W. A. Dudek. S. Mirvakili, Neutral elements, fundamental relations and n-ary hypergroups, Internat. J. Algebra Comput., 19(4) (2009), 567-583.

[7] M. De Salvo, Feebly canonical hypergroups, J. Combinatorics, Inform. \& Syst. Sci., 15 (1990), 133-150.

[8] M. Dresher and O. Ore, Theory of multigroups, Amer. J. Math., 60 (1938), 705-733.

[9] P. Dwinger, Introduction to Boolean Algebras, Wrzburg, (1971).

[10] M. M. Ebrahimi, A. Karimi and M. Mahmoudi, On quotient and isomorphism theorems of universal hyperalgebras, Ital. J. Pure Appl. Mat, 18 (2005), 9-22.

[11] G. Grätzer, A representation theorem for multi-algebras, Arch. Math., 3 (1962), 452-456.

[12] G. Grätzer, Universal Algebra, Second edition, Springer-Verlag, (1979).

[13] G.E. Hansoul, A subdirect decomposition theorem for multialgebras, Algebra Universalis, 16 (1983), 275281.

[14] H. Höft and P. E. Howard, Representing multi-algebras by algebras, the axiom of choice, and the axiom of dependent choice, Algebra Universalis, 13 (1981), 69-77.

[15] M. Koskas, Groupoids, demi-hypergroupes et hypergroupes, J. Math. Pures Appl., 49(9) (1970), $155-192$. 
Alg. Struc. Appl. Vol. 5 No. 1 (2018) 1-21.

[16] V. Leoreanu-Fotea and B. Davvaz, n-hypergroups and binary relations, European J. Combinatorics, 29 (2008), 1207-1218.

[17] V. Leoreanu-Fotea, Contributions to the study of the heart of a hypergroup, Doctoral Thesis: Babes-Bolyai University, Cluj-Napoca, Romania, (1998).

[18] F. Marty, Sur une generalization de la notion de groupe, 8th Congress Math. Scandenaves, Stockholm, (1934) 45-49.

[19] R. Migliorato, On the complete hypergroups, Rivista di Matematica Pura ed Applicata, 14 (1994), 21-32.

[20] C. Pelea, On the fundumental relation of a multialgebra, Ital. J. Pure Appl. Mat., 10 (2001), 327-342.

[21] C. Pelea, Identities and multialgebras, Ital. J. Pure Appl. Mat., 15 (2004), 83-92.

[22] C. Pelea and I. Purdea, Multialgebras, universal algebras and identities, J. Aust. Math. Soc., 81 (2006), 121-139.

[23] H. E. Pickett, Homomorphisms and subalgebras of multialgebras, Pacific J. Math., 21 (1967), 327-342.

[24] S. Rasouli and B. Davvaz, Lattices derived from hyperlattices, Comm. Algebra, 38(8) (2010), 2720-2737.

[25] S. Rasouli and B. Davvaz, Construction and spectral topology on hyperlattices, Mediterr. J. Math., 7 (2010), 249-262.

[26] S. Rasouli and B. Davvaz, Homomorphism, ideals and binary relations on hyper-MV algebras, J. of MultValued Logics \& Soft Computing, 17 (2011), 47-68.

[27] S. Rasouli, D. Heydari and B. Davvaz, $\eta$-relations and transitivity conditions of $\eta$ on hyper-MV algebras, J. of Mult-Valued Logics \& Soft Computing, 15 (2009), 517-524.

[28] S. Rasouli, D. Heydari and B. Davvaz, $\beta$-relations and transitivity conditions of $\beta$ on hyper-BCK algebras, Hacettepe Journal of Mathematics and Statistics, 39(4) (2010), 461-469.

[29] S. Rasouli, D. Heydari and B. Davvaz, An investigation on homomorphisms and subhyperalgebras of $\Sigma$ hyperalgebras, Hacettepe Journal of Mathematics and Statistics, 43(6) (2014), 971-984.

[30] D. Schweigert, Congruence relations of multialgebras, Discrete Math., 53 (1985), 249-253.

[31] Y. Sureau, Contribution a la thorie des hypergroupes et hypergroupes operant transivement sur un ensemble, Doctoral Thesis, (1980).

\section{S. Rasouli}

Department of Mathematics

Persian Gulf University

Bushehr, 75169, Iran.

srasouli@pgu.ac.ir

\section{B. Davvaz}

Department of Mathematics

Yazd University

Yazd, Iran.

davvaz@yazd.ac.ir 